\title{
A PHOTOGRAPHIC PERIODOGRAM OF THE SUN-SPOT NUMBERS
}

A. E. DOUGLASS

Reprinted for private circulation from

The Astrophysical Journal, Vol. XI, No. 3 , October IgI 4 


\section{A PHOTOGRAPHIC PERIODOGRAM OF THE SUN-SPOT NUMBERS}

A. E. DOUGLASS

Reprinted for private circulation from

The Astrophysical Journal, Vol. XL, No. 3, October igi4 


\title{
A PHOTOGRAPHIC PERIODOGRAM OF THE SUN-SPOT NUMBERS
}

\author{
By A. E. DOUGLASS
}

In I898 Professor Arthur Schuster suggested the construction of a periodogram which should display rhythmic time intervals in any series of measures, in the same manner that a spectrogram shows the space vibrations or waves in a beam of light. He analyzed the sun-spot numbers as an example, and showed how several periods, $4.38,4.80,8.36$, II.I25, and I3.5 years, seemed to be operating, ${ }^{\mathrm{I}}$ some more intensely than others.

In I9I 3 Kimura $^{2}$ performed an analysis of the same by a method of successive approximations and derived the amplitudes of a large number of different periods, which when combined produce a result remarkably like the sun-spot curve since $175^{\circ}$. Some of his chief periods are: $82.2,54.2,20.03$, I 2.05 , II.II4, I0.48, 9.99, and 8.55 years. Last year also Professor Turner took up the problem with consummate skill and untiring energy. ${ }^{3}$ On thorough mathematical analysis in the first three papers, he finds that the main features of the sun-spot record can be represented by four periodic terms of approximately 8.3, I0.2, II.4, and I4.7 years, but that their coefficients do not remain constant, and only the II.4-year period is sensible at the present time. Meanwhile A. A. Michelson ${ }^{4}$ applied the harmonic analyzer and determined the amplitudes of a considerable number of periods, such as 8.6,

I "On the Investigation of Hidden Periodicities, with Application to a Supposed 26-Day Period of Meteorological Phenomena," Terrestrial Magnetism, 3, I3-4I, I898; "On the Periodogram of Magnetic Declination at Greenwich," Cambridge Philosophical Society Transactions, 18, I07-135; "The Periodogram and Its Optical Analogy," Proceedings of the Royal Society, 77 A, I36-I40, I906; "On the Periodicities of SunSpots," Philosophical Transactions of the Royal Society of London, 206 A, 69-100, I906.

${ }_{2}$ "On the Harmonic Analysis of Sunspot Relative Numbers," Monthly Notices, R.A.S., 73, 543, г913.

3 Monthly Notices, R.A.S., 73, 549, May I913 (discussion of Kimura's paper); 73, 714, sup. number, I913 (applying the Fourier sequence); 74, I6, November I913 (continuing the last); $\mathbf{7 4}, 82$, December r9r3 (discussing discontinuities and the meteoric hypothesis).

4 "Determination of Periodicities by the Harmonic Analyzer with an Application to the Sun-Spot Cycle," Astrophysical Journal, 38, 268, 1913. 


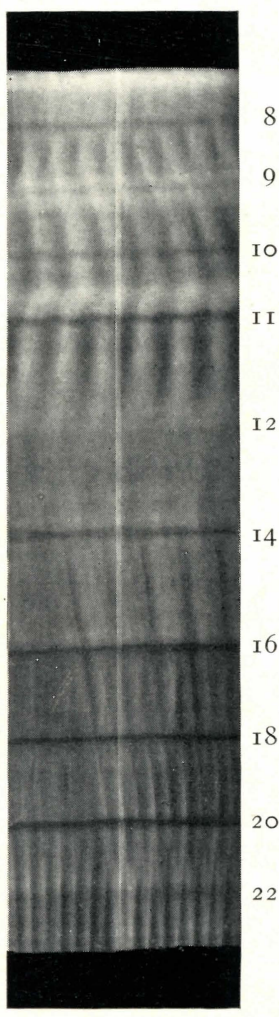

FIG. I.-Periodogram of the sun-spot numbers. Corrugations show periods. The numbers give time of vibrations in years. The white line is the year 1830 and shows phase.

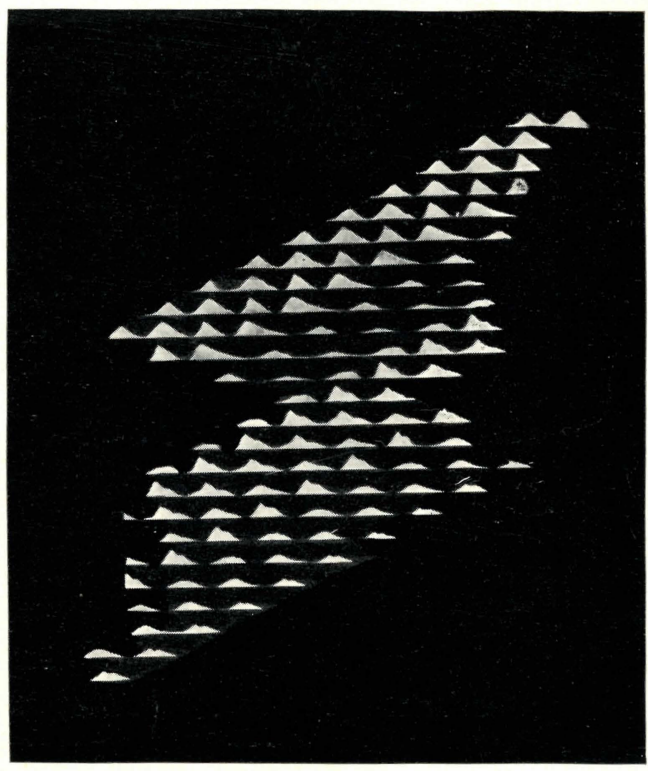

FIG. 2.-Diagram used in making the periodogram, consisting of the sun-spot curve mounted in multiple.

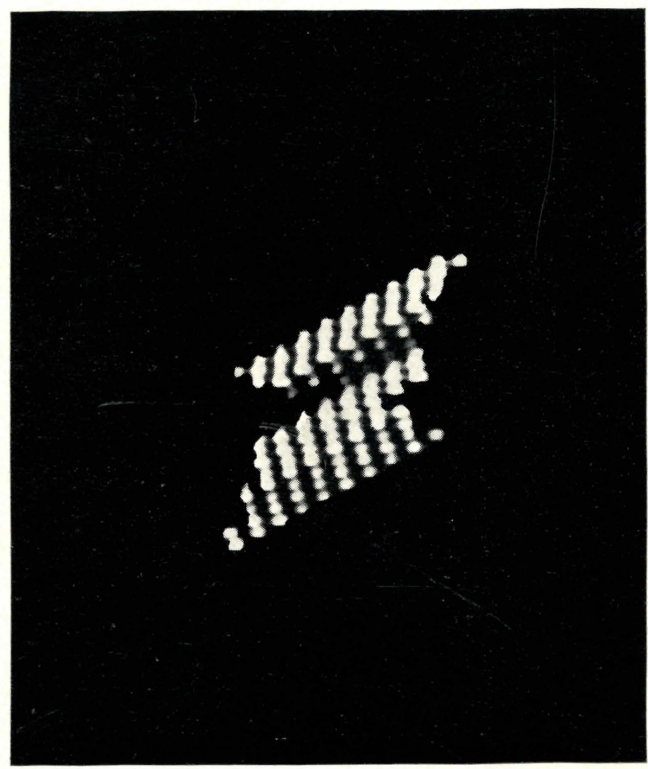

FIG. 3.-Figure 2 photographed out of focus to show discontinuities in the vertical lines. 
I0.3, II.4, I5. I, 58, and I05 years, but most of these appear to him doubtful. He concludes, "Indeed it would seem that with the exception of the I I-year period and possibly a very long period (of the order of roo years), the many periods found by previous investigators are illusory."

But Turner in his last article (December I9I3) presents the matter from a new viewpoint. He investigates discontinuities in the series and finds that the breaks in the sun-spot series "are near the dates I766, г796, г838, г868, I895, which are sufficiently close to those of Leonid periphelia to suggest a vera causa," and he discloses his hypothesis of the meteoric origin of sun-spot periodicity, a hypothesis of the greatest interest but not pertinent to the present subject.

The work described below adds little to the information obtained in the investigations alluded to, but it presents the sun-spot history in a new way and suggests perhaps a rapid means of carrying on preliminary studies of periodicity in any series of continuous records.

In Plate XIV, Fig. I is a periodogram of the sun-spot curve from I755 to I9II, made by a photographic process in which the camera has done the additions for all the periods named beside it. The existence of a rhythm in any specified period is indicated by a beaded or corrugated effect. The corrugations are in fact the rhythmic vibrations of the curve. On a moment's examination this periodogram shows much of the information referred to above. The II-year period is the most pronounced, yet not so superior to all others as would be expected. It may be of any duration from II to II.8 years; II. 4 is a good average. There is obviously a period somewhere between 9.5 and 10.5 years and one between 8.0 and 8.8 , but it is less conspicuous. Faint indications of periods are found near I4 years. The double of 8.4 is seen between I 6 and I 7 years. The double of the ro-year period shows near the 20 , and at 22 the double of the I I begins. Other photographs made at the same time ${ }^{\mathrm{I}}$ show the same periods extremely well, but were less satisfactory in some of the mechanical or photographic details.

${ }^{x}$ May i9r3. H. H. Clayton had produced a periodogram by mechanical means some years previously. The writer here wishes to acknowledge his indebtedness to Professor E. C. Pickering for permission to use some of the facilities at Harvard College Observatory in this work.

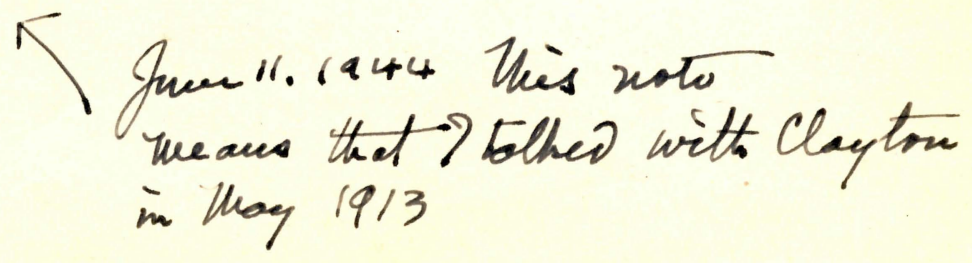


In all cases the phase is located by the central white line which represents very closely the year 1830 . In this photo-periodogram therefore, we have at once pictured to the eye some of the general results of the mathematical work. The method has the disadvantage that we cannot get absolute amplitudes, but perhaps some photometric work could give approximate values if it were worth trying. But relative amplitudes show at once; and we can judge, not only of the relative importance of different periods, but of the precision, or its lack, with which any period can be stated.

Fig. 2 shows a part of the process. It is the diagram from which the periodogram was photographed. In order to produce it the sun-spot curve was cut out in white paper and pasted in multiple, as is seen, on a black background. The left end of each of the upper to lines is the date I755. Each successive line is moved to years to the left, so that passing from above vertically downward each successive line represents a date ro years later than its predecessor. This continues until the whole period from I755 to I9I I is covered; and the lower ro lines show the latter date at their right ends. It is not necessary that any of the lines should be full length, as we use only a part of each. Now, by passing the eye downward from the top, a period near Io years will show itself at once by the successive crests in vertical alignment. If the crests form a line at some angle to the vertical, then the period they indicate is not exactly ro years; it is more than ro if the slant is to the right and less than Io if the slant is to the left. The horizontal lines are spaced the equivalent of 5 years, hence if we measure the angle A made by a vertical line and a line joining two crests in successive horizontal lines, we find the period indicated is

$$
P=\text { Io years }+5 \tan A
$$

where $A$ is positive if measured on a slant to the right.

Since each angle with the vertical represents a different period, it was only necessary to mount this diagram on an axis with clock work and slowly rotate it, in front of a camera with a cylindrical lens over its objective and a narrow horizontal slit in the focal plane and a sensitive plate passing slowly downward across the slit, to produce the periodogram. The cylindrical lens with 
horizontal axis summates the duplicate curves in whatever line happens to be vertical. Of course there is a practical limit to the different angles at which the diagram may be viewed. An angle too far in one direction, making the tested period very small, would require a great number of duplications of the curve, while too great an angle the other way, making the tested period very large, catches the curve used here in a non-symmetrical form and introduces error. In the periodograms actually made of the sunspot curve the minimum period tested was 7 years and the maximum 24 years. One notes especially that this is a continuous process and that all periods from the minimum to the maximum are tested.

In the arrangement described above, there are several limitations to the accuracy of results. First, the curves are nonsymmetrical about their horizontal bases, and, when summated. in a slant line far from the vertical, crests are thrown to one side of their proper place. Plotting the curves above and below a line would improve the result. Secondly, the resolving power decreases as the slant either way increases: the plot must be on larger and larger scale to overcome this. Thirdly, the wider the slit in the focal plane, the less the resolving power. Fourthly, there is a photographic limitation due to the failure of the photograph to show slight contrasts, and, fifthly, the eye cannot ordinarily detect contrasts under about ro per cent.

When one applies this method as here described to other curves, a new condition presents itself, for the percentage variation in most curves is far less than in the sun-spot numbers. One must therefore cut off the lower part of the plot between the zero line and the lower extremes of the curve; for example, it would be thus in a series of barometer readings. However, in spite of these limitations the photographic periodogram does give important information quickly and plainly to the eye and at little cost, and thus can serve as a guide, showing when more refined methods are desirable.

But in common with any single mathematical treatment, the periodogram has one defect, it assumes the variables to be continuous throughout the series. It seems to the writer that Turner 
has caught an important secret of the sun-spot problem, namely, that the variables are discontinuous. One can see from his papers how great a labor it has taken to reach that conclusion. This accounts for much of the discordance between investigators and the disappointment one has felt in the lack of definite result and a basis of prediction for the future.

Now it would take several periodograms of the type here presented to show this discontinuity, but the method here given, with a slight change, does show the whole history of the sun-spot discontinuities at a glance. Fig. 3 is a photograph of Fig. 2, taken out of focus for the purpose of calling attention to certain general features. In Fig. 2 the eye naturally turns to the sharp outlines and notes its minute details. In Fig. 3 the crests of 2 are changed into large blotches connecting somewhat with their nearest neighbors and varying in intensity. The sun-spot sequence appears in each nearly vertical line of blotches. Having a number of exactly similar lines side by side, the irregularities are repeated in each and thus strike the consciousness with the effect of repeated blows. These irregularities are the discontinuities referred to by Turner in connection with his hypothesis. It is evident at a glance that the sun-spot sequence divides itself into three parts, namely, a 9.3-year period, I750-I790, then an interval of readjustment, I800-I830, with a I3-year period, and lastly an II.I-year period lasting to the present time (values approximate). ${ }^{\mathrm{r}}$ But the latter is not perfectly constant, for after 1870 there is a change in intensity. The breaks thus shown and Turner's dates of discontinuity are here compared.

\begin{tabular}{|c|c|c|}
\hline & Periodogram & $\begin{array}{r}\text { Turner } \\
\text { I } 766\end{array}$ \\
\hline Between & I 788 and I804. & . I 796 \\
\hline " & I830 and I837. & .1838 \\
\hline “ & I870 and I884 . & . . 1868 \\
\hline
\end{tabular}

${ }^{I}$ In discussing the periodicities of sun-spots (pp. 75-78) Schuster divided his I 50 years, from 1750 to 1900 , into two nearly equal parts. He found in the first part two periods of $9^{\frac{1}{4}}$ and $\mathrm{I}^{\frac{3}{4}}$ years acting, successively, and in the second part, a period of I I. I years. This is shown graphically in Fig. 3 . 
By means of this diagram, one can discover at a glance the origin of many of the periods which Michelson thought were illusory, and in which he was largely right. We can plainly see a 9.3-year period in the early part of the curve; let us call this part of the sequence $A_{n}$; and let us call its broken continuation near the center $B_{n}$, and the lower and later part giving the II.I-year period, $C_{n}$. Thus we get at once three periods 9.3 , II.I, and something over I 3 years. If now we bring the average $A_{n}$ into line with the average $C_{n}$ as the periodogram does we get II.4 years. If we bring the average $A_{n}$ into line with the average $C_{n-\text { r }}$ we get close to Io years. If we bring into line $A_{n}$ and the heavier parts of $C_{n-2}$ we get 8.4 years or thereabouts. And at 5.6 years we find a period which is just half of $C_{n}$ and at 4.7 we find the half of $A_{n}$, and so on. It is like a checker board of trees in an orchard; they line up in a number of directions with more or less intensity. But the diagram in Fig. 3 helps remove some of the complexity of the sun-spot problem. It shows us that while these various periods are apparent, yet many are illusory as Michelson said. The diagram supplies a basis for profitable judgment in the matter. Hence to avoid just such awkward cases as the sun-spot curve, it is here presented as a necessary accompaniment of the periodogram in any doubtful cases.

University OF ARIZONA

July I9I4 